\title{
Observer training for computer-aided detection of pulmonary nodules in chest radiography
}

\author{
Diederick W. De Boo • François van Hoorn • \\ Joost van Schuppen - Laura Schijf • \\ Maeke J. Scheerder • Nicole J. Freling • Onno Mets • \\ Michael Weber • Cornelia M. Schaefer-Prokop
}

Received: 26 September 2011 /Revised: 16 November 2011 / Accepted: 9 December 2011 /Published online: 25 March 2012

(C) The Author(s) 2012. This article is published with open access at Springerlink.com

\begin{abstract}
Objectives To assess whether short-term feedback helps readers to increase their performance using computer-aided detection (CAD) for nodule detection in chest radiography. Methods The 140 CXRs (56 with a solitary CT-proven nodules and 84 negative controls) were divided into four subsets of 35; each were read in a different order by six readers. Lesion presence, location and diagnostic confidence were scored without and with CAD (IQQA-Chest, EDDA Technology) as second reader. Readers received individual feedback after each subset. Sensitivity, specificity and area under the receiver-operating characteristics curve (AUC) were calculated for readings with and without CAD with respect to change over time and impact of CAD.

Results CAD stand-alone sensitivity was $59 \%$ with 1.9 false-positives per image. Mean AUC slightly increased over time with and without CAD ( 0.78 vs. 0.84 with and 0.76 vs. 0.82 without $C A D$ ) but differences did not reach significance. The sensitivity increased $(65 \%$ vs. $70 \%$ and $66 \%$ vs. $70 \%$ ) and specificity decreased over time (79\% vs. $74 \%$ and $80 \%$ vs. $77 \%$ ) but no significant impact of CAD was found.
\end{abstract}

D. W. De Boo $(\bowtie) \cdot$ F. van Hoorn · J. van Schuppen • L. Schijf • M. J. Scheerder $\cdot$ N. J. Freling $\cdot$ O. Mets $\cdot$ C. M. Schaefer-Prokop Department of Radiology, Academic Medical Centre (AMC), Meibergdreef 9,

1105 AZ Amsterdam, The Netherlands

e-mail: d.w.deboo@amc.uva.nl

\section{Weber}

Department of Radiology, General Hospital Vienna,

University Hospital,

Vienna, Austria

C. M. Schaefer-Prokop

Department of Radiology, Meander Medical Center,

Amersfoort, The Netherlands
Conclusion Short-term feedback does not increase the ability of readers to differentiate true- from false-positive candidate lesions and to use CAD more effectively.

Key Points

- Computer-aided detection (CAD) is increasingly used as an adjunct for many radiological techniques.

- Short-term feedback does not improve reader performance with CAD in chest radiography.

- Differentiation between true- and false-positive CAD for low conspicious possible lesions proves difficult.

- CAD can potentially increase reader performance for nodule detection in chest radiography.

Keywords Radiographic image interpretation .

Computer-assisted $\cdot$ Solitary pulmonary nodule .

Radiography $\cdot$ Lung $\cdot$ Education

\section{Introduction}

Various computer-aided detection (CAD) systems for chest radiography with and without FDA approval have been developed. The latest reported stand-alone sensitivities of these systems for the detection of small focal opacities vary from $34 \%$ to $78 \%$, depending on the lesion selection and study group [1-6].

Results of studies evaluating the effects of CAD on actual observers' performances are not homogeneous and range from significant improvement $[2,7,8]$ to lack of any impact [9]. Results seem to be influenced by the type of CAD algorithm used, reader experience and the conspicuity of the study lesions.

The potential of CAD to increase the radiologist's sensitivity for pulmonary nodules was described earlier: two studies reported that $35 \%$ and $47 \%$ of bronchogenic 
tumours missed in the original reports were correctly marked by CAD [1, 4]. A third study found a lower agreement between CAD and observers' detection compared with the agreement between observers indicating the ability of CAD to mark lesions the radiologists tend to miss [3]. All three studies, however, compared the CAD performance with previously made observer readings and did not assess the actual influence of CAD on the readers' decisions.

The importance of this interaction between CAD and the observers for a successful implementation of CAD was shown by de Hoop et al. [9]. They had not been able to show a positive effect of the CAD algorithm they tested because readers had difficulties in differentiating truepositive from false-positive CAD candidates. More than two-thirds of the true-positive CAD candidates in whom $\mathrm{CAD}$ was given for lesions that were originally missed were not accepted by the readers.

The authors suspected that this inability of the readers to use the CAD more beneficially was at least partly due to a lack of experience with the performance of CAD and subsequently a lack of confidence in the $\mathrm{CAD}$ analysis. For $\mathrm{CT}$ colonography, it was reported that a 1-day training period already resulted in increased reader sensitivity, but at the expense of decreased specificity and increased reading time [10]. In mammography sensitivity, specificity and area under the receiver-operating characteristics curve (AUC) significantly increased after a 4-week training period [11]. The ultimate learning curve for CAD in mammography has been estimated to be around 2 years but this has never been evaluated in a structured manner [12]. Up to now there have been no studies evaluating the effect of observer training on the application of CAD in chest radiography.

The purpose of the current study was to test whether short-term feedback to readers on their own performance when using $\mathrm{CAD}$ would increase the readers' confidence in the CAD analysis and thus their ability to differentiate truefrom false-positive CAD candidates.

\section{Materials and methods}

\section{Study population}

For this retrospective study we selected 140 patients from our institution's data archive. Patients were included if a two-view chest radiograph (CXR) and thoracic CT were obtained within 6 weeks and revealed no or a single nodular opacity. The diameter ranged from $5 \mathrm{~mm}$ to $15 \mathrm{~mm}$ (measured on axial CT images) and none of the nodules showed calcifications. Of the 140 patients 56 had a solitary CTproven nodule and 84 served as negative controls. Patients with more than one nodular opacity or a pathological feature other than COPD on the CXR were excluded.
Ethics committee approval was obtained and because of the retrospective nature of the study patient informed consent was waived (registration no. 10171150).

Pulmonary nodules

Thoracic CT served as a reference standard and revealed a solitary nodular opacity in 56 patients $(40 \%)$. Conspicuity of the lesion on the CXR was subjectively graded in consensus by a board-certified chest radiologist ( $>15$ years of experience) and the researcher (fifth year resident) who were not involved in the readings and ranged from high (1) to moderate (2), low (3) and very low (4).

Image acquisition

All CXRs were obtained using a digital technique with a dedicated chest stand (Thoravision Philips Medical Systems, Hamburg, Germany). Images were processed using non-linear multifrequency processing (Unique, Philips Medical Systems, Hamburg, Germany). Processing parameters were implemented following the recommendations of the manufacturer and represented the same as those used as standard processing in our institution. Both the posteroanterior and the lateral views were available for evaluation.

\section{CAD}

We used a commercially available CAD system (IQQAChest; EDDA Technology, Princeton Junction, NJ, USA). This system is designed to detect nodules within the range from $5 \mathrm{~mm}$ to $15 \mathrm{~mm}$ in diameter on the PA radiograph. Images are automatically analysed in the background after acquisition of the images; thus results are immediately available when the radiographs are read but are only shown on demand. The CAD algorithm marks between zero and five suspicious areas with semitransparent circles (candidates).

Image evaluation

Six observers of vastly varying experience participated in this study: five radiology residents with 0 to 5 years of training (R1, 0 years; R2 and R3, 2 years; R4, 3 years; R5, 5 years) and one board-certified radiologist (R6) with more than 15 years' experience in reading chest films. Two observers (R1 and R3) had no previous experience at all with using $\mathrm{CAD}$ in chest radiography; the other four observers had served as observers in previous studies evaluating CAD, two using the same CAD system as in this study and two using a different CAD system. None of the observers had experience with CAD in clinical routine work.

The 140 pairs of PA and lateral radiographs were divided into four subsets of 35 each. Each subset consisted of 14 
cases with a solitary nodular opacity and 21 negative control cases. Care was taken that the distribution of nodule conspicuity and the CAD stand-alone sensitivity were equal for the four subsets.

Each observer individually interpreted the $35 \mathrm{PA}$ and lateral chest radiographs of one subset first without and subsequently with the availability of the CAD markings within a single reading session. Observers were asked to determine the presence or absence of an intrapulmonary opacity using a five-point scale of confidence ranging from 5 (pulmonary nodule definitely present) to 3 (equivocal with respect to the presence of a nodule) and 1 (definitely no nodule present). For a confidence rating $>1$, observers were asked to indicate the anatomical location of the suspected lesion on a separate data sheet. Readings with and without the availability of the CAD results were separately documented. Readers were allowed to modify their confidence levels after CAD also became available for lesions seen during unassisted reading. The observers were informed that images contained only a single nodular opacity and were instructed to ignore calcified lesions and lesions smaller than $5 \mathrm{~mm}$ in diameter. Magnification, window/level adjustment and grey-scale reversal were allowed during both readings with and without $\mathrm{CAD}$ results.

All observers read the four subsets of CXRs in a different order.

Feedback on readers' performance

After completion of each of the four subsets of cases, the observer received individual feedback on his/her performance by the researcher. During this feedback, the observer and researcher discussed, on a case by case basis, the location of the lesions if present, the CAD marks with respect to whether they were true- or false-positive and the observer's individual response.

\section{Data analysis}

The stand-alone performance of CAD was determined by calculating the sensitivity and mean false-positive per image (mFP). A one-way ANOVA with Tukey post hoc test was used to test differences among the four subsets.

Sensitivity, specificity and AUC were calculated per observer and per subset for the readings with and without the availability of CAD results. For calculating the sensitivity, ratings $1-3$ were considered negative, and ratings 4 and 5 were considered positive if the lesions were correctly localised.

The literature is controversial with regard to whether application of CAD as a second reader should allow for the discharge of lesions located during primary unassisted reading (as done in our study) or should only be used to add potential lesions (add-on mode) [13]. We therefore also analysed the data for the add-on scenario with preservation of all originally indicated lesions. Comparisons of all three methods were made using Cochran Q tests as well as logistic regressions for repeated measurements (GEE). Pairwise comparisons were carried out using McNemar's test for each reader separately. To assess the impact of the feedback on reader performance we compared the results of all readers for the first two subsets with the results for the last two subsets.

All analyses were performed in SPSS 17. Statistical significance was assumed at $P<0.05$.

\section{Results}

\section{Study group}

Patient mean age was 61 years with no significant difference among the four subsets (59.7, 58.8, 61.9 and 60.3). All subsets consisted of 14 patients with a solitary nodule and 21 negative control patients. Of all nodules $30 \%$ (17) had a high conspicuity, $23 \%$ (13) a moderate, $30 \%$ (17) a low and $16 \%$ (9) a very low conspicuity (Table 1). None of the patients of the diseased or of the control group showed any relevant pathological feature other than the effects of smoking and the focal study lesion.

\section{CAD stand-alone}

Stand-alone CAD detected 32 out of 56 nodules leading to a mean sensitivity and sensitivity per subset of $57 \%$. Sensitivity was $100 \%, 54 \%, 44 \%$ and $22 \%$ for nodules of high, moderate, low and very low conspicuity, respectively. CAD generated a total of $260 \mathrm{FP}$ candidates with an mFP of 1.9. The $\mathrm{mFP}$ for the different subsets amounted to 1.6, 2.0, 1.5 and 2.3 with a significant difference between subsets 3 and 4 $(P=0.024)$. The positive predictive value was $0.31(32 / 103)$, whereas the negative predictive value was $0.35(13 / 37)$.

Reader performance for subsets 1 and 2

Sensitivity, specificity and AUC were not significantly affected by the use of CAD (Table 1). Mean AUC increased

Table 1 Distribution of false-positive (FP) computer-aided detection (CAD) candidates and nodule conspicuity per subset

\begin{tabular}{llllll}
\hline Subset & Total CAD & \multicolumn{3}{l}{$\begin{array}{l}\text { Nodule } \\
\text { conspicuity }\end{array}$} \\
& FP & 1 & 2 & 3 & 4 \\
& & 5 & 4 & 4 & 4 \\
A & 56 & 3 & 4 & 3 & 3 \\
B & 70 & 4 & 4 & 5 & 4 \\
C & 52 & 2 & 2 & 2 & 3 \\
D & 82 & & & & \\
\hline
\end{tabular}


Table 2 Averaged reader performance for subsets $1+2$ and $3+4$ without and with CAD. Confidence intervals are shown in parentheses

\begin{tabular}{lllllll}
\hline & Sensitivity & & Specificity & & AUC \\
\hline & $1+2$ & $3+4$ & $1+2$ & $3+4$ & $1+2$ & $3+4$ \\
No CAD & $65 \%(58-73 \%)$ & $70 \%(63-77 \%)$ & $79 \%(74-84 \%)$ & $74 \%(69-80 \%)$ & $0.76(0.71-0.81)$ & $0.82(0.78-0.86)$ \\
$\begin{array}{l}\text { CAD with possible } \\
\text { discharge }\end{array}$ & $66 \%(59-73 \%)$ & $70 \%(63-77 \%)$ & $80 \%(75-85 \%)$ & $77 \%(72-82 \%)$ & $0.78(0.73-0.83)$ & $0.84(0.79-0.88)$ \\
CAD add-on & $69 \%(62-76 \%)$ & $71 \%(65-78 \%)$ & $76 \%(71-81 \%)$ & $74 \%(69-80 \%)$ & $0.78(0.73-0.83)$ & $0.85(0.82-0.89)$ \\
\hline
\end{tabular}

$C A D$ computer-aided detection

$A U C$ area under the receiver operating characteristics curve

CAD with possible discharge: observers were allowed to discharge lesions made during primary unassisted reading

CAD add-on: observers were only allowed to add potential lesions during the reading with $\mathrm{CAD}$

from 0.76 without $\mathrm{CAD}$ to 0.78 with $\mathrm{CAD}$, but the difference did not reach significance.

Use of CAD in the add-on scenario led to an increase in sensitivity ( $65 \%$ vs. $69 \%$ ) but at the expense of loss of specificity ( $79 \%$ vs. $76 \%$ ). If discharge of lesion candidates was allowed with the use of CAD, sensitivity changed from $65 \%$ to $66 \%$ and specificity from $79 \%$ to $80 \%$. None of the differences listed reached statistical significance.

\section{Reader performance for subsets 3 and 4}

Reading sessions 3 and 4, sensitivity, specificity and AUC were not significantly affected by the use of CAD (Table 2). Readers increased their baseline sensitivity to a mean of $70 \%$ at the expense of slightly lower specificity of $74 \%$ compared with the first two reading sessions. With CAD the AUC slightly increased from 0.82 to 0.84 , but the differences did not reach significance.

While sensitivity remained unchanged with CAD (70 and $71 \%$, respectively), specificity slightly increased with CAD when discharge of lesions was allowed (77 and $74 \%$, respectively). None of the differences listed reached statistical significance.

\section{Reader-CAD interaction}

Analysing pooled data, all six readers dismissed $17 \%$ of the TP CAD candidates $(32 / 192)$. There was no difference between the first two and last two subsets (16 vs. 16). Of the dismissed TP candidates $50 \%(16 / 32)$ referred to nodules of low conspicuity and $28 \%(9 / 32)$ to lesions of very low conspicuity with the remaining $22 \%$ (7/32) referring to moderately conspicuous nodules. The number of dismissed true-positive CAD amounted to 1/1, 0/0, 6/4, 1/0, 1/0 and 1/ 1 for subsets 1 and 2 versus subsets 3 and 4 , respectively for readers 1 to 6 .

Based on pooled data analysis the number of accepted FP CAD candidates non-significantly decreased from a total of ten made in the first two readings to six made in the last two readings. An example of reader-CAD interaction is given in Fig. 1.

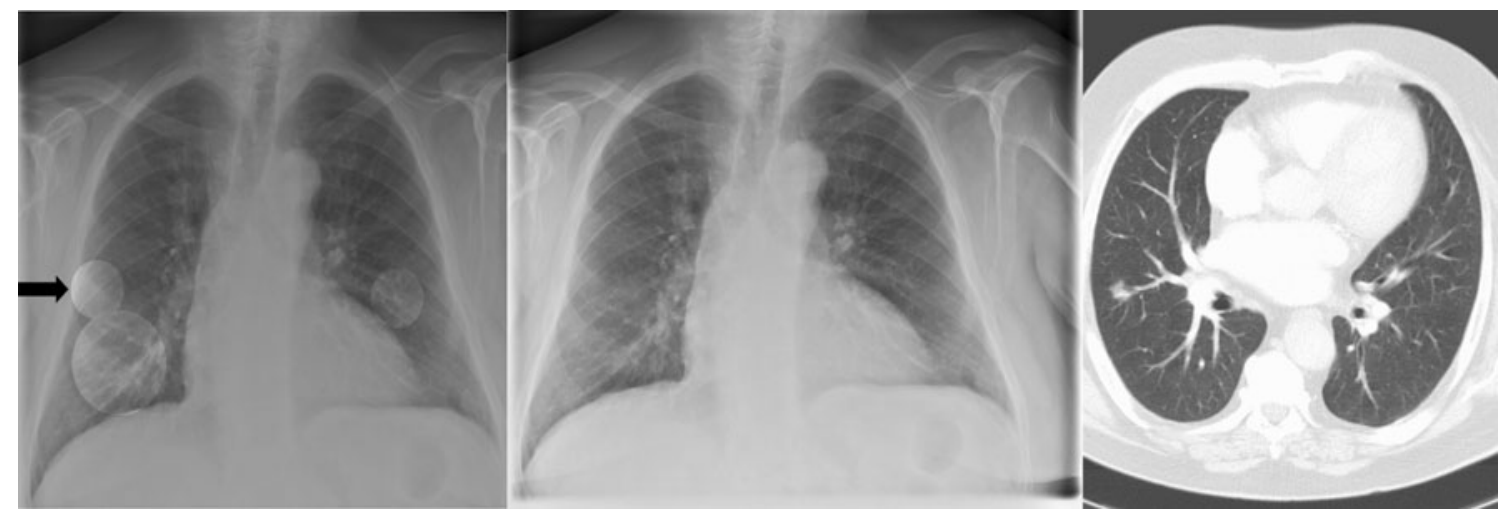

Fig. 1 The nodule of low conspicuity in the right lower lobe was missed by three readers without the computer-aided detection (CAD) results. The true-positive $\mathrm{CAD}$ candidate was accepted by two readers and dismissed by one reader (arrow). None of the readers accepted the false-positive CAD candidates 


\section{Discussion}

It is common clinical practice that small primary lung carcinomas are missed on two-view chest radiographs although they were frequently visible in retrospect. Miss rates of between $20 \%$ and $90 \%$ have been reported for primary lung carcinomas [14-20]. Two recent papers reported a CAD stand-alone sensitivity of $35 \%$ and $47 \%$ for pulmonary tumours initially overlooked by the radiologist [1, 4], indicating the potential of CAD to improve the readers' detection performance. Both papers, however, did not include observer performances; thus, to which extent radiologists would have taken advantage of CAD and accepted these true-positive candidates remains unanswered. Equally the risk of accepting false-positive candidates with unnecessary follow-up diagnostics cannot be quantified. The importance of the interaction between the CAD and observer has been previously illustrated by a study that tested the impact of CAD on observer performance for the detection of T1 tumours on chest radiographs of patients who had been part of a CT screening trial: the number of malignancies initially not seen by the observers but correctly annotated by CAD varied between 5 and 16 per observer. However, $80 \%$ of these correctly annotated lesions (46/59) were not accepted by the readers and subsequently dismissed [9]. One underlying reason for the readers' inability to differentiate true- from false-positive lesions might have been the lack of experience with the $\mathrm{CAD}$ algorithm and subsequently insufficient trust in its performance.

In the current study we therefore tested the effect of short-term feedback on the detection of pulmonary nodules on digital chest radiography. Our hypothesis was that individual feedback after the interpretation of each of the four subsets would help readers to build up more confidence in the performance of the CAD algorithm, eventually resulting in an increased ability to distinguish true- from falsepositive candidates and in a higher acceptance of truepositive CAD candidates.

We found a slight but not significant increase in baseline performance between sessions 1 and 2 compared with sessions 3 and 4, meaning that there was a small overall training effect and that readers detected more nodules in the last two readings than in the first two readings. For none of the paired sessions, however, could we prove a significant influence of reader performance by CAD. Neither the acceptance of true-positive CAD candidates nor the ability to dismiss false-positive candidates was significantly influenced by the feedback information. There was an overall tendency towards increased performance with CAD but the differences were too small to reach significance.

Seventy-eight percent of the dismissed true-positive CAD candidates referred to lesions of low and very low conspicuity, indicating that readers had difficulties in assigning sufficient credibility to CAD candidates, indicating nodules with low conspicuity. Feedback did not help in this respect because the number of dismissed true-positive candidates was the same in sessions 1 and 2 compared with sessions 3 and 4 .

There are very few studies in the literature evaluating the impact of training on the use of CAD and those referred for CT colonography and mammography. Results were quite different in the respect that a short, 1-day period of training already affected observer performance in CT colonography as opposed to mammography, which had a lower learning curve requiring at least 4 weeks $[10,11]$. It seems that the effect of CAD follows different perception and learning rules in CT vs. radiography, and that the beneficial use of CAD to reduce perception errors of well-defined and more conspicuous lesions (e.g. colon polyps) can be learned faster than the use of CAD to detect lesions of low conspicuity that require differentiation from obscuring background noise (e.g. lesions on mammography). This is also supported by our result that most of the dismissed true-positive CAD candidates referred to lesions of low and very low conspicuity.

It is very likely that lesions of high or moderate conspicuity that have been missed by the readers owing to "inattentional blindness" meaning that the lesions missed during routine reporting take advantage of the availability of CAD more effectively and more easily. Unfortunately it is much more difficult to prove this effect under study conditions because readers tend to analyse the radiographs with an especially high degree of alertness compared to normal conditions. Even though the readers in our study generally had a low level of experience in reading chest radiographs5 out of 6 were residents - the baseline sensitivity was relatively high with a mean of $65 \%$ for the first two subsets and $70 \%$ for the last two subsets. It is possible that this high baseline sensitivity impeded a further increase in sensitivity with the availability of CAD results.

Lesions of low or very low conspicuity, however, have different diagnostic requirements: correct diagnosis requires not only visual localisation but also correct differentiation from surrounding "anatomical" noise. For this type of lesion, our results suggest that CAD has no significant impact on reader behaviour and short-term feedback has no effect. Whether a longer learning period would be more efficient as postulated for mammography [12] remains to be proven for chest radiography.

Our results also underline the need to further decrease the number of false-positive CAD candidates. A lower number of false-positive candidates will not only decrease reading time but also increase readers' confidence in the reliability of CAD, and will help them to focus on the presence of underlying lesions in the circled areas of interest. The number of false-positive calls provoked by CAD was quite low in this study: 10 and 6, respectively, pooled over all readers for the first two and last two readings. 
It might also be the case that the pure presentation of CAD candidates alone is not sufficient and more information is needed to help the reader to correctly differentiate true- from false-positive lesions. In this context the availability of likelihood calculations together with an active localisation procedure by the reader him/herself has been found to be very effective for mammography [21].

Our study suffers from the following limitations. Nodule incidence in the study population was higher than usually seen under clinical conditions. Although the readers did not know the exact distribution of positive and negative cases, they certainly were more alert to detecting focal lesions than in a usual clinical setting.

The number of CAD false-positive candidates was not equally distributed over the four subsets, although we consider it unlikely that this had an effect on our results as all readers interpreted the subsets in a different order and there was a generally low number of accepted CAD false-positive candidates.

This study used a specific type of CAD algorithm. It has to be noted that these results are not necessarily transferrable to other CAD algorithms: a different or updated CAD algorithm with a different performance may yield different results in a context in which perception, reader experience and confidence as well as lesion conspicuity form a complicated framework.

Our study represents the experience of one institution; whether a different reader group or involving readers from various institutions would have yielded different results remains speculative.

We conclude that short-term feedback does not significantly increase the ability of readers to differentiate truefrom false-positive candidate lesions in chest radiography in order to use CAD more effectively for the detection of nodular lesions. Further research is needed to determine whether a longer training period or additional processing and display tools such as temporal subtraction, rib suppression or likelihood calculations can increase the benefits of $\mathrm{CAD}$ for reader performance in chest radiography.

Open Access This article is distributed under the terms of the Creative Commons Attribution License which permits any use, distribution, and reproduction in any medium, provided the original author(s) and the source are credited.

\section{References}

1. Li F, Engelmann R, Metz CE, Doi K, MacMahon H (2008) Lung cancers missed on chest radiographs: results obtained with a commercial computer-aided detection program. Radiology 246:273-280

2. Van Beek EJ, Mullan B, Thompson B (2008) Evaluation of a realtime interactive pulmonary nodule analysis system on chest digital radiographic images: a prospective study. Acad Radiol 15:571575

3. Bley TA, Baumann T, Saueressig U et al (2008) Comparison of radiologist and $\mathrm{CAD}$ performance in the detection of CTconfirmed subtle pulmonary nodules on digital chest radiographs. Invest Radiol 43:343-348

4. White CS, Flukinger T, Jeudy J, Chen JJ (2009) Use of a computer-aided detection system to detect missed lung cancer at chest radiography. Radiology 252:273-281

5. Sakai S, Soeda H, Takahashi N et al (2006) Computer-aided detection on digital chest radiography: validation test on consecutive T1 cases of resectable lung cancer. J Digit Imaging 19:376382

6. Hardie RC, Rogers SK, Wilson T, Rogers A (2008) Performance analysis of a new computer aided detection system for identifying lung nodules on chest radiographs. Med Image Anal 12:240-258

7. Xu Y, Ma D, He W (2011) Assessing the use of digital radiography and a real-time interactive pulmonary nodule analysis system for large population lung cancer screening. Eur J Radiol. doi:10.1016/ j.ejrad.2011.04.031

8. Song W, Fan L, Xie Y, Qian JZ, Jin Z (2005) A study of interobserver variations of pulmonary nodule marking and characterizing on DR images. Proc SPIE 5749:272-280

9. de Hoop B, De Boo DW, Gietema HA et al (2010) Computer-aided detection of lung cancer on chest radiographs: effect on observer performance. Radiology 257:532-540

10. Taylor SA, Burling D, Roddie M et al (2008) Computer-aided detection for CT colonography: incremental benefit of observer training. Br J Radiol 81:180-186

11. Luo P, Qian W, Romilly P (2005) CAD-Aided mammogram training. Acad Rad 12:1039-1048

12. Nishikawa R (2007) Increased CAD use prompts look at advantages, drawbacks. Radiological Society of North America Web site. http://www.rsna.org/Publications/rsnanews/feb07/upload/ RSNANews_Feb07_CAD_Usage.pdf. Published February 2007. Last accessed 18 September 2011

13. Giger ML, Chan HP, Boone J (2008) Anniversary paper: history and status of CAD and quantitative analysis - the image role of medical physics and AAPM. Med Phys 35:5799-5820

14. Muhm JR, Miller WE, Fontana RS, Sanderson DR, Uhlenhoop MA (1983) Lung cancer detected during a screening program using 4-month chest radiographs. Radiology 148:609-615

15. Heelan RT, Flehinger BJ, Melamed MR et al (1984) Non-smallcell lung cancer: results of the New York screening program. Radiology 151:289-293

16. Austin JH, Romney BM, Goldsmith LS (1992) Missed bronchogenic carcinoma: radiographic findings in 27 patients with potentially resectable lesion evident in retrospect. Radiology 182:115122

17. Quekel LG, Kessels AG, Goei R, Engelshoven JM (1999) Miss rate of lung cancer on the chest radiograph in clinical practice. Chest 115:720-724

18. Monnier-Cholley L, Arrivé L, Porcel A et al (2001) Characteristics of missed lung cancer on chest radiographs: a French experience. Eur Radiol 11:597-605

19. Shah PK, Austin JH, White CS et al (2003) Missed non-small cell lung cancers: Radiographic findings of potentially resectable lesions evident only in retrospect. Radiology 226:235-241

20. Wu HM, Gotway MB, Lee TJ et al (2008) Features of non-small cell lung carcinomas overlooked at digital chest radiography. Clin Radiol 63:518-528

21. Samulski M, Hupse R, Boetes C, Mus RD, den Heeten GJ, Karssemeijer N (2010) Using computer-aided detection in mammography as a decision support. Eur Radiol 20:2323-2330 